\title{
Fusion-Based Sensor Selection for Optimal State Estimation and Minimum Cost
}

\author{
(Intelligent Optimization Approach)
}

\author{
Saeed Mohammadloo \\ Department of Electrical Engineering, Malek Ashtar University of Technology, Tehran, Iran \\ Email: mohammadloo_s@yahoo.com \\ Ali Jabar Rashidi \\ Vice-chancellor for Research and Technology, Malek Ashtar University of Technology, Tehran, Iran \\ Email: aiorashid@yahoo.com
}

\begin{abstract}
This paper proposes a heuristic method for the sensor selection problem that uses a state vector fusion approach as a data fusion method. We explain the heuristic to estimate a stationary target position. Given a first sensor with specified accuracy and by using genetic algorithm, the heuristic selects second sensor such that the fusion of two sensor measurements would yield an optimal estimation in a target localization scenario. Optimality in our method means that a trade-off between estimation error and cost of sensory system should be created. The heuristic also investigates the importance of proportion between the range and bearing measurement accuracy of selected sensor. Monte Carlo Simulation results for a target position estimation scenario showed that the error in heuristic is less than the estimate error where sensors are used alone for estimation, while considering the trade-off between cost and accuracy.
\end{abstract}

Index Terms - data fusion, sensor selection, multi-objective optimization, genetic algorithm.

\section{INTRODUCTION}

Today target localization by using sensor fusion systems is one of the main issues of tracking scenarios. Estimation accuracy, sensor characteristics and cost of sensory system are three important criteria help for optimal sensor selection in a sensor data fusion problem. Sensor selection system designers are weighting these three criteria and some other factors in every situation.

In a target tracking application, observations about angular direction, range, and range rate are used for estimating a target's position, velocities, and accelerations in one or more axes. The Collected observations from various similar or dissimilar sensors can be used in a sensor data fusion system to make a better decision. Many applications and different methods of sensor data fusion are presented in [1-5].

In all of sensor fusion methods, sensors equipped in the mobile robot or a manufacturing system are used in sensor fusion. However, there are some considerations by using any type of sensors for sensor fusion. For instance, it is desirable to minimize the cost of the sensory system. Therefore, a sensor selection technique before fusing sensor measurements is very useful in order to approve such considerations. Various strategies and approaches have been proposed for the sensor selection problem in [6-9]. A sensor selection algorithm based on the Modified Riccati Equation (MRE) is used to allocate two sensors equipped on a moving platform to track targets in [6]. Another sensor selection technique incorporated with sensor fusion is introduced by Takamasa Koshizen in [7]. The Author has extended The Gaussian Mixture of Bayes with Regularized Expectation Maximization (GMB-REM) as a robot localization system in terms of the external sensor selection task to reach a minimum error in the position of the robot.

This paper is organized as follows. In the next section, we briefly describe the state vector fusion method based on the Extended Kalman Filter that was utilized in our work. We show how two sensor measurements can be fuse to have an accurate state estimation. Section III describes the sensor selection concept. In section IV, we precisely formulate our proposed method for the sensor selection problem. We consider a target localization scenario and describe the sensor selection problem as a multi-objective optimization problem. Section V presents the simulation results from the application of the proposed method to the case of two sensors, and then we provide our conclusions and the efficiency of the proposed method in section VI.

\section{SENSOR DATA FUSION}

The problem of target tracking using measurements from sensors is of considerable interest in many military such as tracking aircrafts, missiles and unmanned aerial vehicles. It is also useful in civil applications such as robotics, air traffic control, air surveillance, and ground vehicle tracking. A total idea of two sensor data fusion is depicted for target tracking in Fig. (1). 


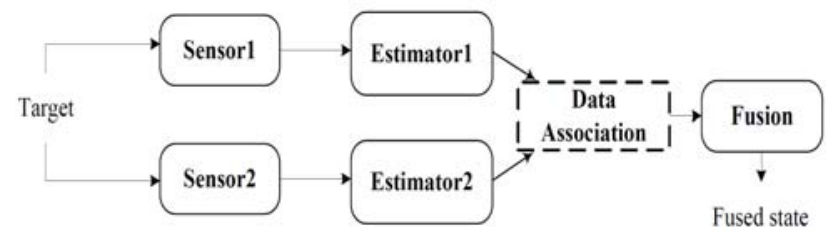

Figure 1. Concept of two sensor data fusion for target tracking [5]

There are generally two broad approaches for the fusion of data: measurement fusion and state-vector fusion. With the former approach, the measurements are basically combined without much processing, and the optimal state vector of target position is obtained. Statevector fusion is preferable in practical situations that the volume of data to be transmitted to the fusion center would be very large. In such a system, each sensor uses an estimator that obtains an estimate of the state vector and its associated covariance matrices (of the tracked target) from the data of that associated sensor. Then these state vectors are transmitted over a data link to the fusion center [5].

Suppose that for a given target tracking scenario, we have an autonomous robot that two sensors such as sonar with different accuracy are installed on it. Using each of these sensors, we would have two estimations from the target states with known and different accuracy. The state vector fusion problem in this scenario will be a weighted sum of the two independent state estimates. Fused state and covariance matrix are computed using the following expressions:

$$
\begin{aligned}
& \hat{X}^{f}=\hat{X}^{1}+\hat{P}^{1}\left(\hat{P}^{1}+\hat{P}^{2}\right)^{-1}\left(\hat{X}^{2}-\hat{X}^{1}\right) \\
& \hat{P}^{f}=\hat{P}^{1}-\hat{P}^{1}\left(\hat{P}^{1}+\hat{P}^{2}\right)^{-1} \hat{P}^{1}
\end{aligned}
$$

Where, $\hat{X}^{1}$ and $\hat{X}^{2}$ are the estimated state vectors of estimator 1 and sensor 2 with measurements from sensor 1 and sensor 2 , respectively, and $\hat{P}^{1}$ and $\hat{P}^{2}$ are the corresponding estimated state error covariance.

\section{SENSOR SELECTION}

In sensor management and sensor data fusion concepts, sensor selection is usually based on the accuracy and performance of each sensor. Although this accuracy is based on certain environmental and sensor related parameters, it does not necessarily mean that the sensor with the highest accuracy also yields the best performance in a sensor data fusion system. With an incorrect sensor selection scheme, it is probable that the fused estimate error be more than the achieved estimation error by each of the sensors.

Another issue here is a cost-benefit tradeoff. In practical situations, usually designers try to obtain the best performance against minimum cost of a sensory system. Also in tracking scenarios, the sensor selection process would be performed based on sensory characteristics. For example, for a given target tracking scenario, one is able to identify the best sensor with a high accuracy in range measuring, in the meantime the other sensor might be used for exact bearing measurements.

\section{Problem Statement}

The proposed algorithm for sensor selection is based on state vector fusion. For a given stationary target, an optimal state estimation is achievable if we minimize the fused covariance $\hat{P}^{f}$. It is obvious from the (2) that the fused state estimate $\hat{X}^{f}$ is directly relative to the fused covariance. Therefore, minimum fused covariance yields an accurate state estimation.

We state the following assumptions:

1) We have a robot equipped with a sensor that has a known performance, named sensor 1 .

2) A stationary target is placed in an unknown location that is able to be found with sensor 1 .

3) The initial position of the robot is available.

\section{A. Target Position Initialization}

Position estimate of the target and its covariance must be properly accomplished for sensor selection. The full set of two dimensional kinematic model of a robot is given by:

$$
\begin{aligned}
& \dot{\psi}=\omega_{z b}+w_{z} \\
& \dot{v}_{x i}=\left(f_{x b}+w_{x}\right) \cos \psi-\left(f_{y b}+w_{y}\right) \sin \psi \\
& \dot{v}_{y i}=\left(f_{x b}+w_{x}\right) \sin \psi-\left(f_{y b}+w_{y}\right) \cos \psi \\
& \dot{x}_{i}=v_{x i} \\
& \dot{y}_{i}=v_{y i}
\end{aligned}
$$

$\omega_{z b}$ is the measured angular rate and $f_{x b}$ and $f_{y b}$ are the measured specific force in body axes of the robot. $w_{x}$, $w_{y}$ and $w_{z}$ are dynamic noise of internal sensor measurements. $v_{x i}$ and $v_{y i}$ are the robot velocity and $x_{i}$ and $y_{i}$ are the position of the robot in a space-fixed reference frame [10].

The robot is equipped with a range/bearing sensor. Laser range finders and sonar sensors are two examples of range/bearing sensors that might use on the robot. Given the robot position $\mathrm{X}_{R}=\left(x_{R}, y_{R}\right)$ and its orientation $\psi$, the observation of range, $z_{r}$ and bearing, $\mathrm{z}_{\theta}$ can be modeled as:

$$
Z=\left[\begin{array}{l}
z_{r} \\
z_{\theta}
\end{array}\right]=\left[\begin{array}{l}
\sqrt{\left(x_{R}-x_{t}\right)^{2}+\left(y_{R}-y_{t}\right)^{2}} \\
\arctan \left(\frac{\left(y_{R}-y_{t}\right)}{\left(x_{R}-x_{t}\right)}\right)-\psi
\end{array}\right]+\left[\begin{array}{l}
w_{r} \\
w_{\theta}
\end{array}\right]
$$

where $w_{r}$ and $w_{\theta}$ are uncertainty in sensor measurements. According to (3)-(7) and (8), the state vector of our system is $\mathrm{X}_{R}=\left[\begin{array}{llllll}x_{R} & y_{R} & \mathrm{v}_{X_{R}} & \mathrm{v}_{X_{R}} & \psi\end{array}\right]$ and observation vector is $\mathrm{Z}=\left[\begin{array}{ll}z_{r} & z_{\theta}\end{array}\right]$.

Given the (8), a target estimation model $g_{t}$, maps the robot position and relative observation to target position estimate. Therefore, the estimate of the target position is: 


$$
\mathrm{X}_{t}=\mathrm{g}_{t}\left(\mathrm{X}_{\mathrm{R}}, \mathrm{z}\right)
$$

and its covariance is :

$$
\mathrm{P}_{t}=A \cdot \mathrm{P}_{0} \cdot A^{T}+B \cdot \mathrm{R} \cdot B^{T}
$$

Where $\mathrm{P}_{0}$ is initial covariance of robot states, $\mathrm{A}$ and $\mathrm{B}$ are Jacobian of $\mathrm{g}_{t}$ evaluated around state vector and observations respectively.

\section{B. Nonlinear Equation System Derivation}

Covariance of observation noise or dynamic noise of measurement for sensor 1 and sensor 2 is defined by $\mathrm{R} 1$ and R2, respectively :

$$
\mathrm{R}_{1}=\left[\begin{array}{cc}
\sigma_{r 1} & 0 \\
0 & \sigma_{\theta 1}
\end{array}\right], \mathrm{R}_{2}=\left[\begin{array}{cc}
\sigma_{r 2} & 0 \\
0 & \sigma_{\theta 2}
\end{array}\right]
$$

where $\left(\sigma_{r}, \sigma_{\theta}\right)$ are standard deviation of range and bearing measurements respectively. According to (8) and (11), we will have two different observation models for sensor 1 and sensor 2 :

$$
\begin{aligned}
& Z_{\mathrm{S} 2}(k)=\left[\begin{array}{l}
z_{r}(k) \\
z_{\theta}(k)
\end{array}\right]+\left[\begin{array}{c}
w_{r_{S 2}}(k) \\
w_{\theta_{S 2}}(k)
\end{array}\right], \\
& Z_{\mathrm{S} 1}(k)=\left[\begin{array}{l}
z_{r}(k) \\
z_{\theta}(k)
\end{array}\right]+\left[\begin{array}{l}
w_{r_{S 1}}(k) \\
w_{\theta_{S 1}}(k)
\end{array}\right]
\end{aligned}
$$

Therefore, covariance estimates for target position by sensor 1 and sensor 2, $\mathrm{P}_{t 1}$ and $\mathrm{P}_{t 2}$, will not have the same values. As we said before, sensor 1 has a known accuracy and is available, hence $\mathrm{R} 1, \mathrm{P}_{t 1}$ and $\mathrm{Z}_{\mathrm{S} 1}$ are non-parametric and take true values. But $\mathrm{R} 2, \mathrm{P}_{t 2}$ and $\mathrm{Z}_{\mathrm{S} 2}$ are parametric values. Further details in the calculation of $\mathrm{P}_{t 2}$ are given in Appendix. Also according to (2), $\hat{\mathrm{P}}^{f}$ will be a $2 \times 2$ parametric matrix based on $\left(\sigma_{r 2}, \sigma_{\theta 2}\right)$.

$$
\hat{\mathrm{P}}^{f}=f\left(\sigma_{r 2}, \sigma_{\theta 2}\right)
$$

The main diagonal of $\hat{\mathrm{P}}^{f}$ is consist of two nonlinear functions. After some simplifications and for a given value of random function, $\hat{\mathrm{P}}^{f}$ takes on the different forms

Now, we try to minimize the fused covariance defined by (13) to have an optimal estimation of target position. Therefore, we can consider the sensor selection problem as a nonlinear multi-objective optimization problem. In other words, $\left(\sigma_{r 2}, \sigma_{\theta 2}\right)$ must be specified in a manner that the partial derivation of (13) goes to zero:

$$
\left\{\begin{array}{l}
E q 1=\frac{\partial^{2}\left(\hat{P}^{f}(1,1)\right)}{\partial \sigma_{r 2} \partial \sigma_{\theta 2}}=0 \\
E q 2=\frac{\partial^{2}\left(\hat{P}^{f}(2,2)\right)}{\partial \sigma_{r 2} \partial \sigma_{\theta 2}}=0
\end{array}\right.
$$

For the above equation set, because of high nonlinearity, it is difficult to calculate $\sigma_{r 2}$ and $\sigma_{\theta 2}$ analytically and the response of equation set is highly correlated to initial values. Fig. (2) and Fig. (3) show the values of specified Eq1 and Eq2 for a given and limit band of $\left(\sigma_{r 2}, \sigma_{\theta 2}\right)$.

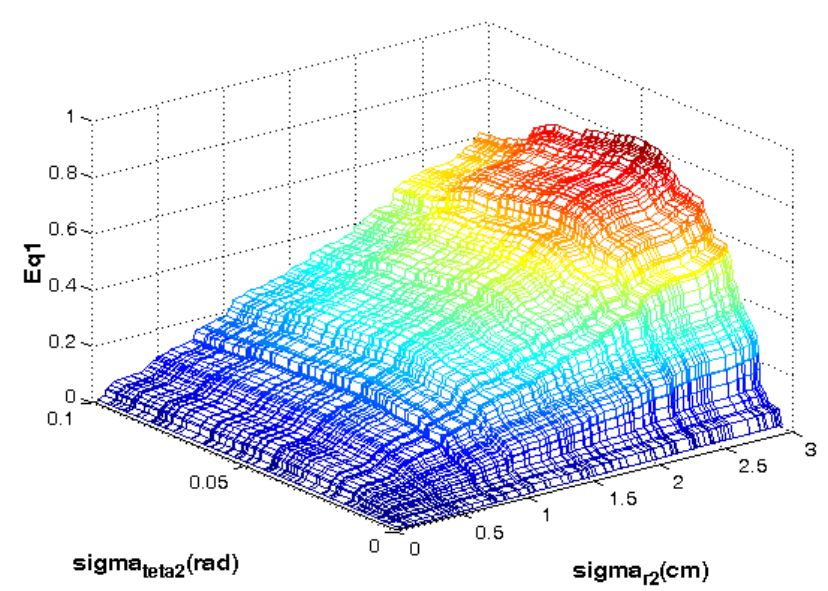

Figure 2. Surface plot of Eq1

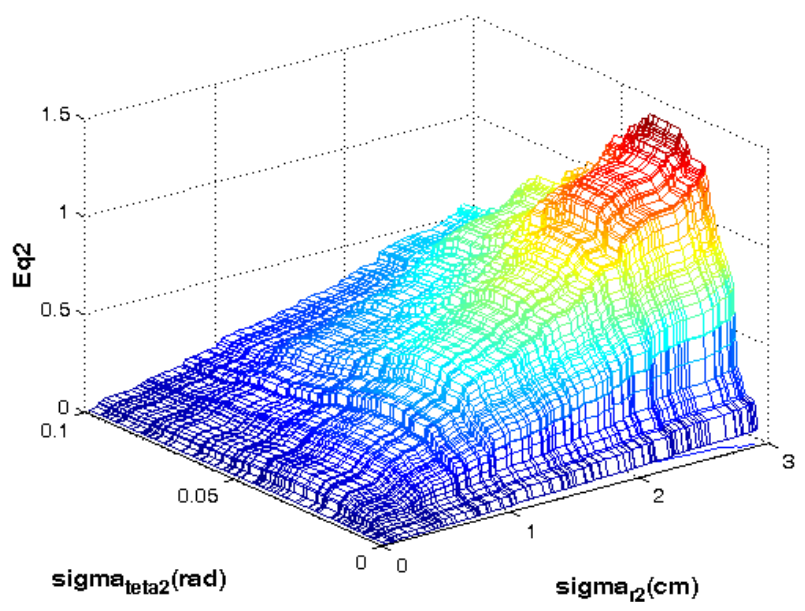

Figure 3. Surface plot of Eq2

Indeed, Fig (2) and Fig. (3) shows variations of original diagonal elements of the fused covariance matrix into $\left(\sigma_{r 2}, \sigma_{\theta 2}\right)$. If we plot these two surfaces in a common frame, some crossing points will appear. It proves that the equation set (16) has more than two answers. Fig. (4) shows these cross points.

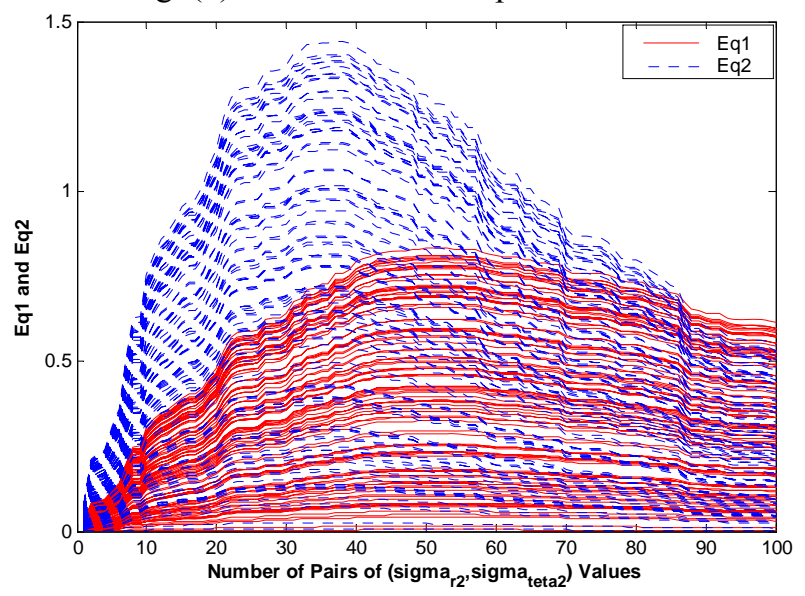

Figure 4. Cross points of surface Eq1 and surface Eq2 in a common frame 
According to the above proofs, we propose multiobjective optimization approaches for solving nonlinear equation sets (14).

\section{B. Transformation into a Multi-Objective Optimization Problem}

The purpose of multi-objective optimization problem is to seek design vector $\boldsymbol{X}^{*}=\left[X_{1}^{*}, X_{2}^{*}, \ldots, X_{n}^{*}\right]^{T}$ according to $k$ object functions $f_{i}$ that must be minimized within a given set of $m$ equality and $p$ inequality constraints that restrict the problem. A mathematical formulation of the multi-objective optimization is :

$$
\begin{array}{ll}
\text { find } & \boldsymbol{X}^{*} \\
\text { optimize } & F(\boldsymbol{X}) \\
\text { subject to } & \left\{\begin{array}{l}
g_{i}(\boldsymbol{X}) \leq 0(i=1,2, \ldots, m) \\
h_{j}(\boldsymbol{X})=0(j=1,2, \ldots, p)
\end{array}\right.
\end{array}
$$

where $X^{*} \in \mathfrak{R}^{n}$ is a design variable vector, $F(\mathbf{X})=\left[f_{1}(X), f_{2}(X), \ldots, f_{k}(X)\right]^{T}$ is object function vector and $g_{i}(X)$ and $h_{j}(X)$ are equality and inequality constraint, respectively. With no loss of generality, we can assume that all of the object functions should be minimized. Such multi-objective minimization problems are classified as Pareto solution problems and for achieving the optimal solution, some definitions such as Pareto front, Pareto set, Pareto optimality and Pareto dominance are used.

In multi-objective problems, heuristic optimization methods, particularly genetic algorithms (GAs) be used extensively during the last decades to search for optimum solutions [11] - [15].

Genetic algorithms which imitate the process of natural evolution have shown successful results in many optimization problems which are difficult to solve by the conventional methods of the mathematical programming. In this work we used Non-dominated Sorting Genetic Algorithm (NSGA-II) for solving systems of nonlinear equations (14) as a multi-objective optimization problem.

The complete NSGA-II procedure is given below:

\section{BEGIN}

While generation count is not reached

Begin Loop

- Create the offspring population $\mathrm{Q}_{\mathrm{t}}$ (of size $\mathrm{N}$ ) using the parent population $\mathrm{P}_{\mathrm{t}}$ ( of size $\mathrm{N}$ ),

- Combine parent $\mathrm{P}_{t}$ and offspring population $\mathrm{Q}_{t}$ to obtain population $\mathrm{R}_{\mathrm{t}}$ of size $2 \mathrm{~N}$.

- Perform Non-dominated Sorting on $\mathrm{R}_{\mathrm{t}}$ and assign ranks to each Pareto front with fitness $\mathrm{F}_{\mathrm{i}}$.

- Starting from the Pareto front with fitness $F_{1}$, add each Pareto-front $F_{i}$ to the new parent population $\mathrm{P}_{\mathrm{t}}+1$ until a complete front $\mathrm{F}_{\mathrm{i}}$ cannot be included.

- From the current Pareto-front $\mathrm{F}_{\mathrm{i}}$, add individual members to new parent population $\mathrm{P}_{\mathrm{t}}+1$ until it reaches the size $\mathrm{N}$.
- Apply selection, crossover and mutation to new parent population $\mathrm{P}_{\mathrm{t}}+1$ and obtain the new offspring population $\mathrm{Q}_{\mathrm{t}}+1$.

- Increment generation count.

\section{End Loop}

END.

\section{Simulation AND Results}

In this section we define the sensor selection problem as a multi-objective optimization problem and we present simulation results to validate the theory developed in the previous sections.

We formulate the multi-objective optimization problem as follows :

$\left\{\begin{array}{l}\text { Optimize } f(x)=\left(f_{1}(x), f_{2}(x)\right) \\ \text { subject to } x=\left(\sigma_{r 2}, \sigma_{\theta 2}\right) \in \Omega\end{array}\right.$

$=\left\{\begin{array}{l}\text { Minimize } \operatorname{abs}\left(f_{1}(x)\right) \\ \text { Minimize } \operatorname{abs}\left(f_{2}(x)\right)\end{array}\right.$

$f_{1}(x)$ and $f_{2}(x)$ in this formulation take the values Eq1 and Eq2 in (14) respectively.

The upper and lower bounds are considered as follows :

Lower Band : $\left[\begin{array}{ll}\sigma_{r 1} & \sigma_{\theta 1}\end{array}\right]$

Upper Band : $\left[n \times \sigma_{r 1} \quad m \times \sigma_{\theta 1}\right]$

where $n$ and $m$ are positive real values.

Again, consider presenting equations in Appendix that are utilized for relative range and bearing $\left(z_{r 2}, z_{t 2}\right)$ calculation between robot and target using sensor 2. As we can see, a random function with zero mean and specified variance $\left(\sigma_{r 1}, \sigma_{\theta 1}\right)$ has been used in these equations. Therefore the performance of proposed method must be evaluated by Monte Carlo simulation. For different random function values, several object functions will be derived and multi-objective optimization algorithm will be served to solve the derived nonlinear equation set.

For simulation, consider the scenario presented in Fig. (5). In this scenario, a target is placed in position $(100,200)$ and we want to estimate the location of the target by a robot that is placed in position $(0,0)$.

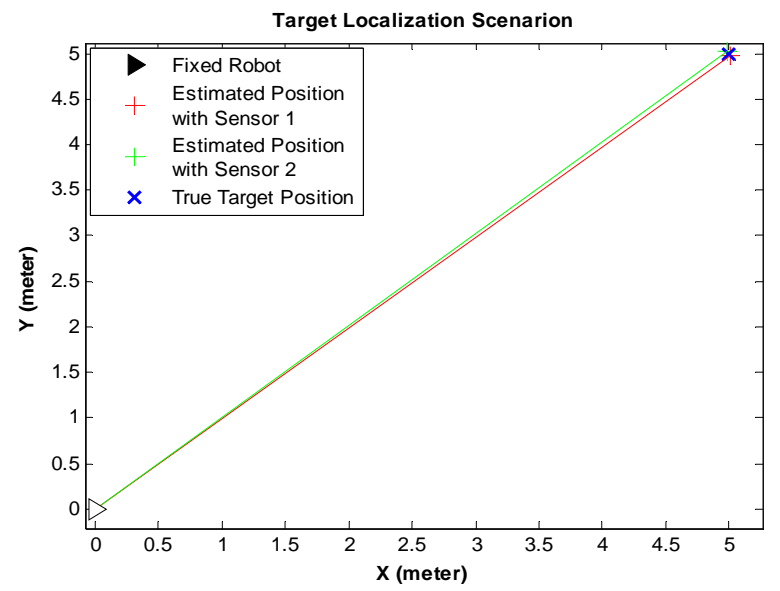

Figure 5. Multi-sensor target localization scenario 
Monte Carlo simulation iterated 100 times. The simulation parameters and characteristics of first sensor are shown in table I. The values of the parameters used in the simulation by genetic algorithm are given in table II.

TABLE I. SIMULATION PARAMETERS AND CHARACTERISTICS OF SENSOR 1

\begin{tabular}{|c|c|c|}
\hline Sampling Period & $\Delta t(k)$ & $0.01 \mathrm{Sec}$ \\
\hline $\begin{array}{c}\text { Noise STD dev in the range } \\
\text { measurement of sensor1 }\end{array}$ & $\sigma_{r 1}$ & $2 \mathrm{~cm}$ \\
\hline $\begin{array}{c}\text { Noise STD dev in bearing } \\
\text { measurement of sensor1 }\end{array}$ & $\sigma_{\theta 1}$ & $0.02 \mathrm{rad}$ \\
\hline Initial position STD dev of Robot & $\sigma_{x}, \sigma_{y}$ & $1 \mathrm{~cm}, 1 \mathrm{~cm}$ \\
\hline Initial velocity STD dev of Robot & $\sigma_{V x}, \sigma_{V y}$ & $1 \mathrm{e}-3 \mathrm{~m} / \mathrm{s}, 1 \mathrm{e}-3 \mathrm{~m} / \mathrm{s}$ \\
\hline Initial heading STD dev of Robot & $\sigma_{\psi}$ & $1 \mathrm{e}-4 \mathrm{rad}, 1 \mathrm{e}-4 \mathrm{rad}$ \\
\hline $\begin{array}{c}\text { Upper and Lower bounds in } \\
\text { optimization algorithm }\end{array}$ & $\mathrm{U}_{\mathrm{b}}, \mathrm{L}_{\mathrm{b}}$ & $5 \mathrm{~cm}, 0.1 \mathrm{rad}$ \\
\hline
\end{tabular}

TABLE II. VALUES OF THE PARAMETERS USED IN THE SIMULATION BY GENETIC ALGORITHM

\begin{tabular}{|c|c|}
\hline Parameter & Value \\
\hline Population Size & 30 \\
\hline Number of Generations & 300 \\
\hline Number of Variables & 2 \\
\hline Function Tolerance & $1 \mathrm{e}-4$ \\
\hline
\end{tabular}

Some of the solutions obtained by a single run and for a given object function as well as the function values (which represent the values of the system's equations obtained by replacing the parameter values) are presented in Table III. The algorithm provides optimal Pareto curve as shown in Fig. (6).

TABLE III. EXAMPLES OF THE SOLUTIONS OBTAINED BY A SINGLE RUN FOR A GIVEN OBJECT FUNCTION

\begin{tabular}{|c|c|c|c|c|c|c|c|c|}
\hline \multirow[t]{2}{*}{.0: } & \multicolumn{2}{|c|}{$\begin{array}{c}\text { Variables } \\
\text { Values }\end{array}$} & \multicolumn{2}{|c|}{$\begin{array}{c}\text { Function } \\
\text { Values }\end{array}$} & \multicolumn{2}{|c|}{$\begin{array}{c}\text { Fusion } \\
\text { Covariance }\end{array}$} & \multicolumn{2}{|c|}{$\begin{array}{c}\text { Target Position } \\
\text { Estimation } \\
\text { Covariance By } \\
\text { Sensor } 2 \\
\end{array}$} \\
\hline & \multicolumn{2}{|c|}{$\sigma_{\theta 2} \sigma_{r 2}$} & \multicolumn{2}{|c|}{ Eq1 Eq2 } & \multicolumn{2}{|c|}{$\hat{P}^{f}(1,1) \hat{P}^{f}(2,2)$} & \multicolumn{2}{|c|}{$\hat{P}^{2}(1,1) \hat{P}^{2}(2,2)$} \\
\hline \multirow{8}{*}{$\begin{array}{c}\text { Sol } \\
1\end{array}$} & 2.930 & 0.028 & $3 e-06$ & 0.0098 & 2.6359 & 2.5393 & 5.1988 & 5.4541 \\
\hline & 3.021 & 0.020 & 0.007 & $1 \mathrm{e}-06$ & 2.612 & 2.5168 & 5.5303 & 5.7221 \\
\hline & 2.934 & 0.021 & $3 e-04$ & 0.007 & 2.6349 & 2.5387 & 5.2274 & 5.4173 \\
\hline & 2.943 & 0.021 & 0.001 & 0.0063 & 2.6325 & 2.5363 & 5.2571 & 5.4514 \\
\hline & 3.000 & 0.020 & 0.005 & 0.0015 & 2.6174 & 2.5219 & 5.4561 & 5.6494 \\
\hline & 2.930 & 0.028 & $3 e-06$ & 0.0098 & 2.6359 & 2.5393 & 5.1988 & 5.4541 \\
\hline & 2.960 & 0.022 & 0.002 & 0.0050 & 2.628 & 2.532 & 5.3133 & 5.5172 \\
\hline & 2.991 & 0.020 & 0.005 & 0.0022 & 2.6198 & 2.5242 & 5.4245 & 5.616 \\
\hline \multirow{8}{*}{$\begin{array}{c}\text { Sol } \\
2\end{array}$} & 3.691 & 0.021 & $4 \mathrm{e}-07$ & 0.028 & 2.345 & 2.470 & 8.626 & 7.484 \\
\hline & 3.090 & 0.055 & 0.068 & $3 e-07$ & 2.458 & 2.599 & 6.584 & 4.610 \\
\hline & 3.090 & 0.055 & 0.068 & $5 e-10$ & 2.458 & 2.599 & 6.584 & 4.610 \\
\hline & 3.122 & 0.021 & 0.025 & 0.003 & 2.454 & 2.587 & 6.246 & 5.429 \\
\hline & 3.094 & 0.025 & 0.031 & 0.001 & 2.460 & 2.594 & 6.187 & 5.251 \\
\hline & 3.319 & 0.021 & 0.013 & 0.015 & 2.412 & 2.542 & 7.027 & 6.103 \\
\hline & 3.416 & 0.022 & 0.009 & 0.020 & 2.393 & 2.522 & 7.437 & 6.430 \\
\hline & 3.082 & 0.031 & 0.040 & $3 e-07$ & 2.462 & 2.598 & 6.220 & 5.083 \\
\hline \multirow{8}{*}{$\begin{array}{c}\text { Sol } \\
3\end{array}$} & 2.903 & 0.057 & $34-10$ & 0.025 & 2.578 & 2.606 & 5.000 & 5.657 \\
\hline & 3.017 & 0.034 & 0.015 & $1 \mathrm{e}-09$ & 2.548 & 2.577 & 5.447 & 5.869 \\
\hline & 2.902 & 0.025 & $4 e-08$ & 0.011 & 2.578 & 2.607 & 5.088 & 5.378 \\
\hline & 3.014 & 0.021 & 0.009 & $1 \mathrm{e}-04$ & 2.549 & 2.578 & 5.484 & 5.743 \\
\hline & 3.017 & 0.026 & 0.012 & $2 \mathrm{e}-05$ & 2.548 & 2.577 & 5.474 & 5.800 \\
\hline & 3.017 & 0.034 & 0.015 & $1 \mathrm{e}-09$ & 2.548 & 2.577 & 5.447 & 5.869 \\
\hline & 2.919 & 0.026 & 0.001 & 0.009 & 2.573 & 2.603 & 5.144 & 5.444 \\
\hline & 2.950 & 0.022 & 0.004 & 0.005 & 2.565 & 2.595 & 5.256 & 5.525 \\
\hline
\end{tabular}

All solutions in a Pareto set are equally optimal. It is up to the designer to select a solution in the Pareto set depending on the application. In this work we choose the mean of solutions as a final answer and select the second sensor based on this answer.

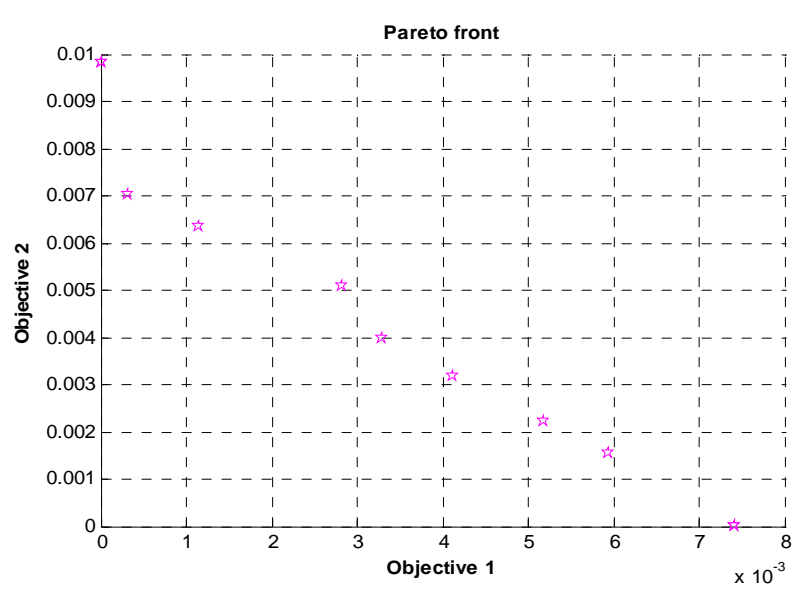

Figure 6. Optimal Pareto solutions for the multi-objective optimization problem

For more analysis, average Pareto distance and spread of generated individuals in genetic algorithm are shown in Fig. (7).
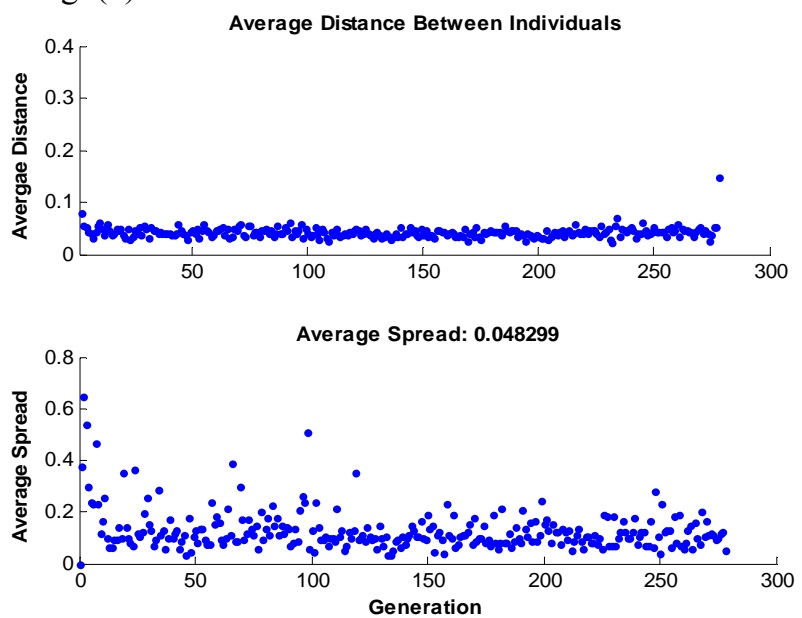

Figure 7. Average Pareto distance and spread of generated individuals

Table IV shows the characteristics of selected sensor. The results are obtained after 100 Monte Carlo simulation runs.

TABLE IV. VALUES OF THE PARAMETERS USED IN THE SiMULATION BY GENETIC ALGORITHM

\begin{tabular}{|c|c|c|}
\hline $\begin{array}{c}\text { Noise STD dev in the range } \\
\text { measurement of sensor 2 }\end{array}$ & $\sigma_{r 2}$ & $3.3939 \mathrm{~cm}$ \\
\hline $\begin{array}{c}\text { Noise STD dev in bearing } \\
\text { measurement of sensor 2 }\end{array}$ & $\sigma_{\theta 2}$ & $0.0396 \mathrm{rad}$ \\
\hline
\end{tabular}

Fig. (8) depicts the time histories of target position estimation errors with $\pm 3 \sigma$ bounds for state vector fusion algorithm scheme when we fuse the obtained measurements by sensor 1 and sensor 2 . Sensor 2 is selected by the proposed sensor selection algorithm. 

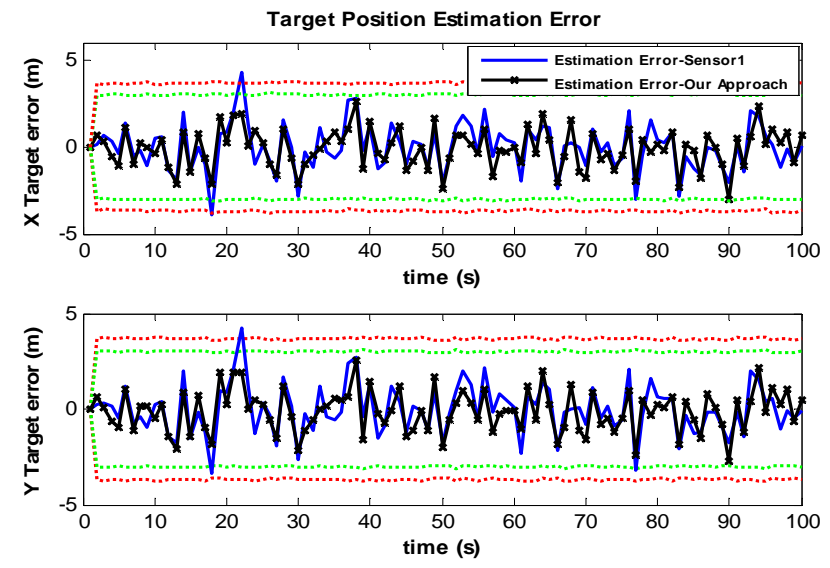

Figure 8. Target position estimation error - sensor 1 and our approach

From the results presented in Fig. (8), one can see that satisfactory accuracy of position estimation has been obtained using the proposed sensor selection algorithm. Fig. (9) presents estimation errors with $\pm 3 \sigma$ bounds when we only use sensor 2 for target position estimation.
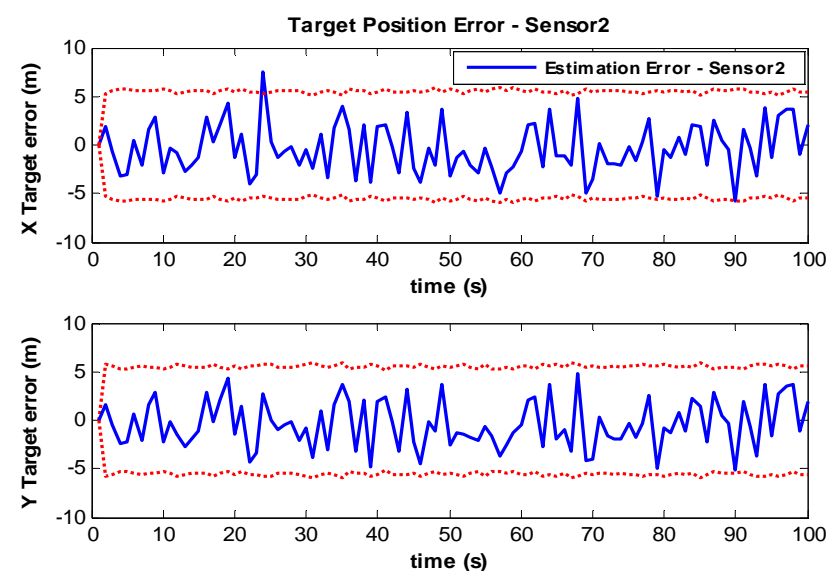

Figure 9. Target position estimation error - sensor 2

Using 100 Monte Carlo simulations, Estimation accuracy was measured by the total RMSE in the position estimates of the target. Results are presented in table V.

\section{TABLE V. VALIDATION RMSE VALUES FOR TARGET POSITION ESTIMATION}

\begin{tabular}{|l|c|c|c|}
\cline { 2 - 4 } \multicolumn{1}{c|}{} & $\begin{array}{l}\text { Estimation } \\
\text { with Sensor 1 } \\
\text { measurements }\end{array}$ & $\begin{array}{l}\text { Estimation } \\
\text { with Sensor 2 } \\
\text { measurements }\end{array}$ & $\begin{array}{l}\text { Estimation with } \\
\text { fused measurements }\end{array}$ \\
\hline Y Position RMSE & 1.4050 & 2.2224 & 1.1037 \\
\hline
\end{tabular}

\section{Discussions AND CONCLUSION}

A new heuristic algorithm for sensor selection problem has been proposed in this paper and its success investigated by some simulations. Given a specified sensor, we selected another one to pair with it. Considering a design area for sensor selection, we defined a multi-objective optimization problem and selected a perfect pair of sensors for optimal state estimation. Using the state vector fusion method, accurate target position estimation performed by the selected sensors such that a trade-off between cost of the sensory system and estimation error is created.

Proposed method gives us more solutions for the sensor selection problem that all of them are optimal, but in this work we choose the mean of solutions to make a sensory system.

It is worth to mention the necessity of using our approach for selection of a perfect pair of sensors in position estimation problems. In section $\mathrm{V}$, we considered a design area for sensor selection. In this section, we ignore our sensor selection algorithm and put the $\left(\sigma_{r 2}, \sigma_{\theta 2}\right)$ equal to upper bound values of design area. Estimation error results with $\pm 3 \sigma$ bounds and total RMSE for this condition are presented in Fig. (10) and table VI respectively.
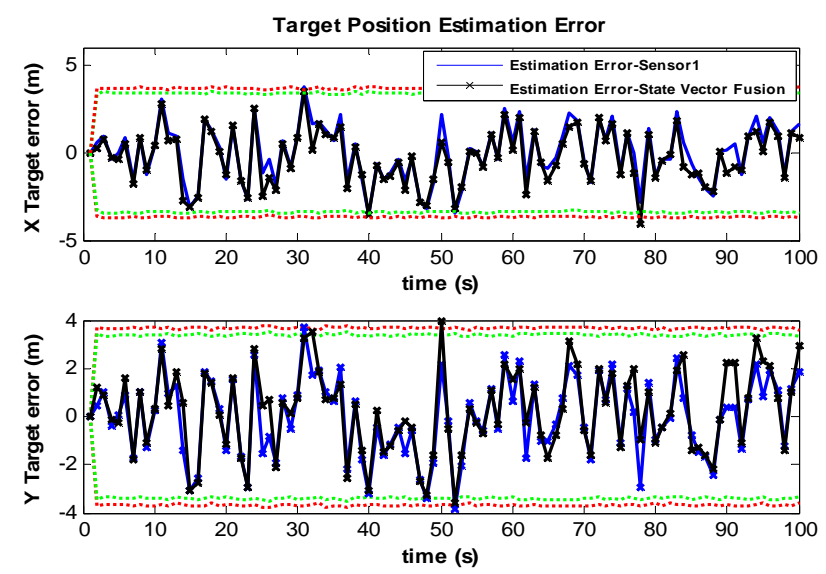

Figure 10. Target position estimation error :

$\left(\sigma_{r 1}=2 \mathrm{~cm}, \sigma_{\theta 1}=0.02 \mathrm{rad}\right)$ and $\left(\sigma_{r 2}=5 \mathrm{~cm}, \sigma_{\theta 2}=0.1 \mathrm{rad}\right)$

TABLE VI. RMSE VALUES FOR TARGET POSITION ESTIMATION : $\left(\sigma_{r 1}=2 \mathrm{~cm}, \sigma_{\theta 1}=0.02 \mathrm{rad}\right)$ AND

$$
\left(\sigma_{r 2}=5 \mathrm{~cm}, \sigma_{\theta 2}=0.1 \mathrm{rad}\right)
$$

\begin{tabular}{|l|c|l|l|}
\cline { 2 - 4 } \multicolumn{1}{c|}{} & $\begin{array}{l}\text { Estimation } \\
\text { with Sensor 1 } \\
\text { measurements }\end{array}$ & $\begin{array}{l}\text { Estimation } \\
\text { with Sensor 2 } \\
\text { measurements }\end{array}$ & $\begin{array}{l}\text { Estimation with } \\
\text { fused measurements }\end{array}$ \\
\hline X Position RMSE & 1.5801 & 7.2762 & 1.9143 \\
\hline Y Position RMSE & 1.5948 & 5.8132 & 1.9626 \\
\hline
\end{tabular}

It is obvious from Fig. (10) and table VI that the state vector fusion algorithm has not successful results for any arbitrary pairs of sensors. While our proposed algorithm always select a perfect pair of sensors such that gives optimal fusion results.

Propriety between the range and bearing measurement accuracy of selected sensor is another important ability of our proposed algorithm. In the state vector fusion algorithm, incorrect selection of sensor 2 may cause to undesired estimation results, while the cost of sensory system is reduced.

For example, consider the presented characteristics of sensor 1 in table I. Here we use a low cost sensor 2 
described by $\sigma_{r 2}=10$ and $\sigma_{\theta 2}=0.001$ in which the propriety between range and bearing measurements is not regarded. In this case, Fig. (11) shows attained estimation accuracy by state vector fusion algorithm.
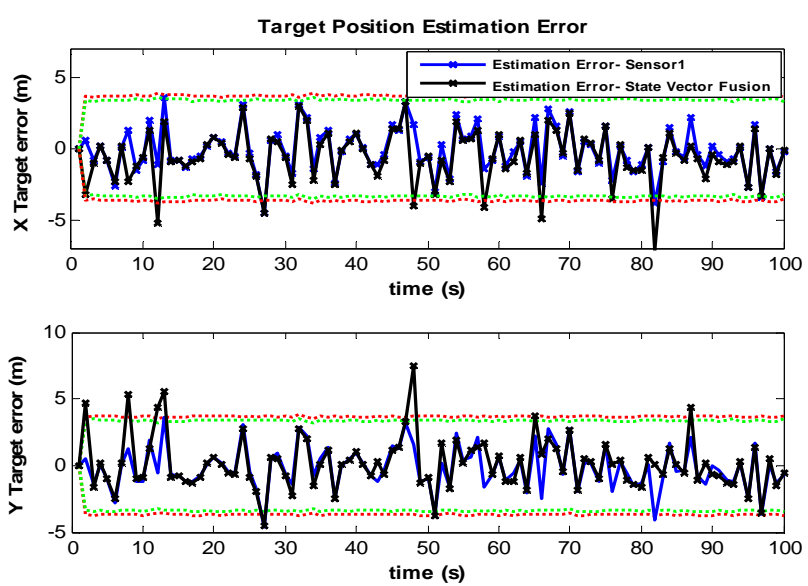

Figure 9. Target position estimation error :

$\left(\sigma_{r 1}=2 \mathrm{~cm}, \sigma_{\theta 1}=0.02 \mathrm{rad}\right)$ and $\left(\sigma_{r 2}=10 \mathrm{~cm}, \sigma_{\theta 2}=0.001 \mathrm{rad}\right)$

As we can see from this figure, fused estimation error is higher, as compare with the case that sensor 1 is used for position estimation, where errors exceed the $\pm 3 \sigma$ confidence bounds.

Our approach is applicable for any type of sensors and selection has done based on the accuracy of sensors in range and bearing measurements. As we know, increasing in measurement accuracy of sensors makes them expensive. Our approach is very valuable for a decision maker to select proper sensor suites according to requirements. Therefore, by utilizing the proposed algorithm, we can select a pair of sensors with minimum cost to have an optimal estimation in target tracking problems.

\section{REFERENCES}

[1] Harmon, S. Y., "Tools for muitisensor data fusion in autonomous robots", Highly Redundant Sensing in Robotic Systems. Springer-Verlag, 1990, pp. 103- 125.

[2] Rashidi, A. J. and Mohammadloo, S., "Simultaneous Cooperative Localization for AUVs Using Range Only Sensors", International Journal of Information Acquisition, 2011, Vol. 8, No. 2, pp. 117 - 132.

[3] Bazzazzadeh, N., "Optimal and Robust Distributed Data Fusion for Joint Target-Detection and Tracking", School of Engineering and Physical Sciences Heriot Watt University, MSc thesis, 2009.

[4] Ir. Nada Milisavljević, "Sensor and Data Fusion", Published by In-Teh, Croatian branch of I-Tech Education and Publishing KG, Vienna, Austria, 2009.

[5] Jitendra R. Raol, "Multi-Sensor Data Fusion with MATLAB", Published by Taylor \& Francis Group, 2010.

[6] Ramdaras, U.D. and Bolderheij, F., "Performance-Based Sensor Selection for Optimal Target Tracking", 12th International Conference on Information Fusion, 2009, pp. 1687-1694.

[7] Takamasa Koshizen, "Improved Sensor Selection Technique by Integrating Sensor Fusion in Robot Position
Estimation", Journal of Intelligent and Robotic Systems, 2000, Vol. 29, Issue. 1, pp. 79-92.

[8] Joshi, S. and Boyd, S. "Sensor Selection via Convex Optimization", IEEE Trans. On Signal Processing, 2009, Vol. 57, Issue. 2, pp. $451-462$.

[9] Yongmian Zhang and Qiang Ji, "Sensor Selection for Active Information Fusion", Proceedings of the 20th national conference on Artificial intelligence, 2005, Volume 3, pp. 1229-1234.

[10] Titterton, D. H. and Weston, J. L. "Strapdown Inertial Navigation Technology," The Institution of Electrical Engineers, 2004.

[11] C. Grosan and A. Abraham, "A New Approach for Solving Nonlinear Equations Systems", IEEE Trans. On Systems, Man and Cybernetics, Part A: Systems and Humans, May 2008, VOL. 38, NO. 3.

[12] K. Deb, S. Agrawal, A. Pratap, T. Meyarivan, "A fast and elitist multi-objective genetic algorithm: NSGA-II" , IEEE Trans. On Evolutionary Computation, 2002, 6(2):182-197.

[13] N. Nariman-zadeh, K. Atashkari, A. Jamali, A. Pilechi, X. Yao, "Inverse modeling of multi-objective thermodynamically optimized turbojet engine using GMDH-type neural networks and evolutionary algorithms", Taylor \& Francis Group, Engineering Optimization, Vol. 37, 2005, pp. 437-462(26).

[14] K. Atashkari, N. Nariman-zadeh, A. Jamali, A. Pilechi, "Thermodynamic Pareto Optimization of turbojet using multi-objective genetic algorithm", International Journal of Thermal Science, Elsevier, 2005, Vol. 44, No. 11, pp. 1061-1071.

[15] N. Srinivas, K. Deb, "Multi-objective Optimization using Non-dominated Sorting in Genetic Algorithm", Journal of Evolutionary Computation, 1994, Vol. 2, No. 3, 221-248.

\section{APPENDIX}

$\mathrm{Z}_{\mathrm{S} 2}=\left[\begin{array}{l}\sqrt{\left(x_{R}(k)-x_{t}(k)\right)^{2}+\left(y_{R}(k)-y_{t}(k)\right)^{2}} \\ \arctan \left(\frac{\left(y_{R}(k)-y_{t}(k)\right)}{\left(x_{R}(k)-x_{t}(k)\right)}\right)-\psi(k)\end{array}\right]$

$+\left[\begin{array}{cc}\sigma_{r 2} & 0 \\ 0 & \sigma_{\theta 2}\end{array}\right] \times \operatorname{Rand}(n)$

$Z_{r 2}=Z_{\mathrm{S} 2}(1)$

$z_{t 2}=Z_{\mathrm{S} 2}(2)$

where $\operatorname{Rand}(\mathrm{n})$ is a function that produces a gaussian white noise sequences. For a given value of this function, main diagonal of $\mathrm{P}_{\mathrm{t} 2}$ takes on the following form :

$$
\mathrm{P}_{t 2}(1,1)=\frac{\sigma_{r 2}^{2}+\sigma_{\theta 2}^{2} \times z_{r 2}^{2} \times \tan ^{2}\left(z_{t 2}\right)}{1+\tan ^{2}\left(z_{t 2}\right)}+0.25
$$

$\mathrm{P}_{t 2}(2,2)=\frac{\sigma_{r 2}^{2} \times \tan ^{2}\left(z_{t 2}\right)+\sigma_{\theta 2}^{2} \times z_{r 2}^{2}}{1+\tan ^{2}\left(z_{t 2}\right)}+0.25$

Saeed Mohammadloo (September 1983), male, Khoy, Iran, research engineer, Malek-e-Ashtar University. His 
research interests are in the areas of autonomous robots, navigation, data fusion, Intelligent Control and Multivariable Control Theory.

Ali Jabar Rashidi received M.A. and Ph.D. degrees from the Tarbiat Modares University in Tehran, Iran in 1997 and 2002, respectively, both in Communication System Engineering. Currently, he is engaged in research and teaching on information fusion, distributed processing, C4I, array sensors and systems, and communications networks. 